\title{
Development of the Motor System: Hopping Rats Produced by Prenatal Irradiation
}

\author{
Samuel P. Hicks and Constance J. D'Amato ${ }^{1}$ \\ Department of Pathology, University of Michigan Medical Center, \\ Ann Arbor, Michigan 48109
}

Received March 6, 1980

\begin{abstract}
Irradiation of prenatal and infant rats resulted in a spectrum of highly reproducible nervous system malformations associated with locomotor abnormalities difficult to correlate with morphologic findings. Fetal rats exposed to $150 \mathrm{R}$ on the 13 th, 14th, or 15th day of gestation were born with a hopping gait, paired hind and forelimbs moving in unison instead of the normal alternating mode. Some animals switched partly or completely to an alternating gait of forelimbs, rarely hind limbs. Rats irradiated on the 12th, 16th, or 17th day did not hop. The problem: Was the hopping related to the brain or spinal cord? Hopping rats could jump to a level or tilted landing platform. Their forelimbs tactually placed independently of each other, whether they hopped or not, but the hind limbs scratched synchronously. Thoracic cord transection led to crossed extension hind-limb reflexes in normal rats, and simultaneous withdrawal of hind limbs in hopping rats, in response to bilateral pinprick. The dorsal horns, especially Rexed's laminae I-VI, and sometimes the most dorsal part of VII, which were being formed in the 13-to 15-day period as shown by tritiated thymidine autoradiography, were underdeveloped. This was due to failure to make restitution of residual dorsal proliferative cells remaining after radiation. Some neurons destined for the dorsomedial parts of the ventral horns may have been lost after the 13th-and 14th-day irradiation, but not the 15th. Precisely how dorsal horn deficiencies could affect the spinal locomotor generator, presumed to be more ventrally situated, it is not yet known. Nor has the exact nature of the suprasegmental adaptation to the hopping mechanism and switching to normal forelimb gait been worked out.
\end{abstract}

\section{INTRODUCTION}

As a basis for this report, we described in the preceding paper the postnatal development of corticospinal (CS) neurons, identified by

Abbreviations: HRP_-horseradish peroxidase, CS-corticospinal.

' This research was supported by U.S. Public Health Service grant NS10531. We thank Lester T. Rutledge for suggestions for neurological testing of the rats. 
retrograde axonal labeling with horseradish peroxidase (HRP) in rats irradiated with $150 \mathrm{R}$ on the 12th, 14th, or 15th prenatal day and with 150 or $200 \mathrm{R}$ on the 17 th day (4). We sought to find how the patterns of cortical development, including that of CS neurons, might be correlated with the development of these animals' motor function. Normally, CS neurons are distributed in a minor rostral band in layer $\mathrm{V}$ of area 10 of the dorsal motor-sensory cortex, projecting to the cervical cord, and a major caudal band approximately in areas 3,4 , and 6 , projecting as far as the lumbar cord. The gap between the bands contained CS neurons projecting to the cervical cord until 2 weeks of age, when the projections disappeared. If the caudal band was partly ablated, the gap projections persisted. Most 12th-day irradiated rats (hereafter 12-day rats, etc.) walked and jumped normally, and they were anatomically normal except for a small reduction in the diameter of the spinal cord in some cases and occasional bifurcation of apical dendrites of CS neurons, despite the initial destructive effects of radiation. Seventeen-day rats usually walked with an abnormal rhythm of cyclic locomotor limb movements, which in a greater or lesser degree they were unable to adapt to difficult terrain such as a narrow path. The gait suggested a disorder of cortical-striatal-thalamic-cortical loop circuits important in programming, learning, and controlling movement $(1,17,19)$. Some rats exposed to $200 \mathrm{R}$ also had difficulty placing their feet on narrow paths during locomotion (impaired locomotor placing), reminiscent of the effects of ablating cortical areas 3, 4, and 6 . Those exposed to $150 \mathrm{R}$ were able to jump variable distances and directions, visually guided (4). Seventeen-day rats had much reduced, scrambled dorsal cortices including areas 3,4 , and 6 and especially area 10 rostrally. CS neurons in reduced numbers were present in the caudal band, gap, and rostral band, but they were often displaced and disoriented. Aberrant bundles of fibers, many of thalamic origin, laced the cortex $(10,15)$. Because area 10 was the chief source of corticostriate projections in the rat, in contrast to areas 3,4 , and 6 , the principal source of CS fibers and projections to the posterior column nuclei, a close correlation between malformed cortex and abnormal motor function appeared possible. Ablation of dorsal area 10 did not appreciably affect locomotion, however; it was therefore suggested that the malformation of dorsal area 10 with its abnormal wiring, especially abnormal thalamocortical fibers, as well as the deficits, might explain the disordered locomotion.

Fourteen- and 15-day rats were characterized by a hopping gait, a thin cortex with an extensive subcortical ectopia, and deficient and disordered development of the posterior gray matter of the cord. Caudal and rostral bands and gap contained subnormal numbers of CS neurons. There were CS neurons in the subcortical ectopia, distributed in a mirror image of those 
in the overlying cortex. The bizarre gait in these rats was attributed to the spinal cord abnormality $(10,15)$ or to disorder of brain mechanisms for alternating limb movement (18). To better understand the hopping form of locomotion, we studied aspects of the developing motor function, certain reflexes, and the morphology of the spinal cord in rats exposed to $150 \mathrm{R}$ on the 14th or 15 th prenatal day. We also made some comparisons between these rats and others irradiated on the 12th or 17th prenatal day, and with normal controls. Preliminary reports of our data have been made $(9,11)$.

\section{METHODS}

Methods were those previously described, with some additional procedures (4). Essentially, the rats were the F1 cross (Irish) between black-selfed nonagouti males and albino Wistar females, both from long brother-sister-mated lines, and occasionally a line of hooded rats derived from the Irish. For study of developing motor behavior, the normal and variously irradiated rats were introduced to broad and narrow, level, horizontal wood pathways (tracks) and equal-level jumping platforms, all elevated, at about 2 weeks of age, where they first crawled, then walked, or hopped, on the paths and stepped short distances, then jumped progressively longer distances on the platforms. The platform for jumping was round; that for landing, rectangular. In some experiments the landing platform was tilted sideways about $40^{\circ}$ to test adjustment of the limbs on landing. Reinforcement was the opportunity to explore and to be handled by the observers (8). One broad path was $13 \mathrm{~cm}$ wide, $2.7 \mathrm{~m}$ long, and slightly curved, and the other straight, $150 \mathrm{~cm}$ long, and $9 \mathrm{~cm}$ wide. The principally used narrow path was $13 \mathrm{~mm}$ wide, $2 \mathrm{~cm}$ high, and $150 \mathrm{~cm}$ long. Paths were marked at intervals of 5 or $10 \mathrm{~cm}$. Slow-motion movies (64 frames per second) recorded the animals' various activities. A mirror $150 \mathrm{~cm}$ long and slightly inclined behind each track made it possible to photograph both sides and limb pairs of the animals simultaneously in many movies. In others the four limbs were photographed from one side. Gait diagrams (16) and measures of consecutive strides of the two hind limbs (4), speed, and other features of movement were made from a frame-by-frame analysis of the movies. Movies were also made of normal and 14- and 15-day rats being dropped from the supine position at about $30 \mathrm{~cm}$ to compare their righting movements. Some 15-day rats were introduced as adults to horizontal ladders, about $12 \mathrm{~cm}$ wide, to see whether or not locomotion on these tracks altered hopping. The rungs on one ladder were rectangular, $1 \mathrm{~cm}$ in diameter, and spaced $3.5 \mathrm{~cm}$ on centers; the rungs of the other were round dowels $6 \mathrm{~mm}$ in diameter spaced $1.8 \mathrm{~cm}$ on centers. The 14- and 15-day rats were also tested in swimming, in a $60-\mathrm{cm}$ square tank of water at about $38^{\circ} \mathrm{C}$. 
Toward finding out whether the hopping was generated in the spinal cord or in supraspinal centers, three 15-day hopping rats and three normal rats, as adults, had a surgical transection of the midthoracic spinal cord with removal of a segment 1 or $2 \mathrm{~mm}$ long, under ether anesthesia. Responses to pin prick or touch of the dorsum or plantar surface of one or both hind feet simultaneously, and to pinching one foot or both feet, were observed from shortly after the operation to 2 to 4 months. The animals' bladders were emptied by manual pressure for the first few days; thereafter they emptied automatically. Conduct of all experiments, use of anesthesia in the performance of surgical procedures, and care of the animals conformed to the standards of the U.S. Department of Health, Education and Welfare Publication (NIH) 78-32, Guide for the Care and Use of Laboratory Animals, and the American Association for Accreditation of Laboratory Animal Care.

Anatomic and regular histologic studies of the animals as a whole, and their brains and spinal cords, and methods for labeling CS neurons were described previously $(3,4)$. Posterior root ganglia at various levels of some 12-, 14-, and 15-day rats were compared with those of normal animals. It was difficult to measure such variably shaped structures and the survey was inconclusive. A more reliable method using earlier stages is being attempted. For histologic examination, the spinal cords were prepared appropriately and stained with cresyl violet, luxol fast blue with cresyl violet or hematoxylin, hematoxylin and eosin, and Cajal's pyridine silver nitrate for nerve cells. To compare the cords from animals irradiated at the various stages, we commonly used the $\mathrm{C} 4$ to $\mathrm{C} 5$ and L1 levels, corresponding to the beginning of the respective intumescences, and other levels including thoracic and sacral were examined. To provide background for development of the spinal cord in relation to the effects of radiation, we have drawn on our continuing study of the normal development of the rat's central nervous system, and some of the early cellular effects of irradiation on the developing nervous system, using tritiated thymidine (H3T) histologic autoradiography. Methods and some results of the study have been previously described $(7,12,14)$.

Rats pregnant on one of the gestational days 11 to 22 were given $\mathrm{H} 3 \mathrm{~T}$ in solution ( $4 \mu \mathrm{Ci} / \mathrm{g}$ body weight; sp act, $3 \mathrm{mCi} / \mathrm{mmol} ; 1000 \mu \mathrm{Ci} / \mathrm{ml}$ ) either by the intracardiac route, when a very short pulse was desired to study stages of mitotic cycles, or intraperitoneally to provide a longer pulse for labeling substantial cohorts of cells being produced on a given day. From two to four successive samples of the litter of fetuses, usually two animals in each sample, were made by surgical removal, including such as $0.5,4,12,24,48$, 72,120 , or more hours after H3T; or two prenatal samples were taken and the remaining fetuses were carried to term and examined in infancy and 
maturity. In some short-pulse experiments radiation was given at various half-hourly or hourly intervals after $\mathrm{H} 3 \mathrm{~T}$ administration to determine what stages of the cell cycle were vulnerable to destruction and what cells contributed to the early restitution of the damage $(12,14)$.

Serial sections of whole, paraffin-embedded younger fetuses, the brains with the base of the skull and upper spine with the first two segments of the cervical cord of perinatal animals, and the brains and upper cervical cords of mature animals were made in sagittal and frontal planes. Fluid emulsion autoradiography (Kodak NTB2 Nuclear Track emulsion) was carried out on interval, or sometimes all, serial sections and counterstained with cresyl violet or acidified (red) Mayer's hematoxylin. Cells whose nuclei contained H3T in a given section were classed as heavily labeled, moderately heavily labeled, or lightly labeled. The more heavily labeled cells were regarded as products of a single, possibly sometimes two, cell divisions after the initial uptake of H3T and considered to represent those cells that originated during the period when the H3T was available. A cell's label depended not only on the number of times it had been approximately halved by mitosis, but also on the duration of the DNA synthesis (S) phase in relaton to the availability of $\mathrm{H} 3 \mathrm{~T}$ and the size of the nucleus in large and small neurons, glia, and their precursors (7).

The positions of the labeled cells en route from their origins to their destinations were plotted from the sequences of stages studied. Although individual cells could not be traced through their migrations, strong inferences about the courses taken by cohorts of labeled cells and their transit times could be made from viewing their positions in a succession of stages and their relations to other labeled and unlabeled cells. In the cord, proliferation of future nerve cells was essentially completed during the 16th and 17th fetal days, and although other attributes of growth continued to progress into mature life, the opportunity to see multiple stages during intrauterine life provided useful information. Our views of the final positions of neurons in mature stages were limited to the cervical cord and were essentially similar to those found at these end stages by others (20).

\section{RESULTS}

\section{Development of Motor Function}

Hopping and Other Synchronous Movements. Beginning at about the time of birth, hind- and forelimb pairs of rats irradiated on the 14th or 15th day moved synchronously rather than in the normal alternating mode. This could be discerned against the background of apparently random movements during the first 2 days by holding up the fore or hind part of the infant's body to emphasize crawling. Of the 19 members of a first group of 

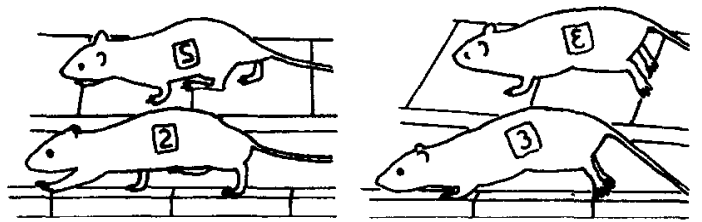

Fig. 1. A hopping rat (right) is compared with a normal rat in motion on a broad track marked every $10 \mathrm{~cm}$ and photographed with a mirror behind the track. Outline tracings of single movie frames.

14- and 15-day rats that grew to adulthood, 6 retained the synchronous hopping movement of hind and forelimbs (Figs. 1, 2), and 8 switched the forelimb pattern to essentially normal alternating locomotion between about 4 and 6 weeks, but hopped with the hind limbs, the forelimb change occurring during several days. Three others mixed alternating and hopping locomotion of the forelimbs, but hopped persistently with the hind limbs; one occasionally hopped with the hind limbs, but otherwise used an alternating gait, and another switched to a continuous virtually normal gait, with rare hops. The latter rat changed to near-normal during the fourth week. Three rats that retained hind- and forelimb hopping were quite clumsy when they came to the edges of flat tracks and platforms, and on the narrow paths had considerable difficulty, but improved somewhat with time. The other rats did about as well on the narrow paths as on the flat paths, retaining the same locomotor patterns, but at a slower pace. In a second group of 10 15-day rats, presently under observation, all have retained hopping locomotion, some displaying mixed hopping and alternate gait in the forelimbs.

Several 15-day hopping rats were introduced to the horizontal ladders as adults. At first they had difficulty traversing these tracks, their limbs slipping between the rungs, but after several minutes they adapted to the terrain and hopped virtually perfectly on the rungs, adjusting their footfalls and strides very well to the two sets of rungs. The hopping locomotion was not dissolved into an alternating gait or otherwise visibly changed.

All adult 14- and 15-day rats were able to swim, their limb movements in water corresponding to those that the individual rat exhibited on tracks. The three most incapacitated animals occasionally became completely immersed momentarily, but resumed head-out swimming quickly. All tactually placed laterally with a forelimb, even those that hopped with the forelimbs; the tested limb moved independently of the other. A contrasting observation made innumerable times was that when rats that hopped with the hind limbs attempted to scratch themselves with one hind limb, the other hind limb moved synchronously with it. [This was also 


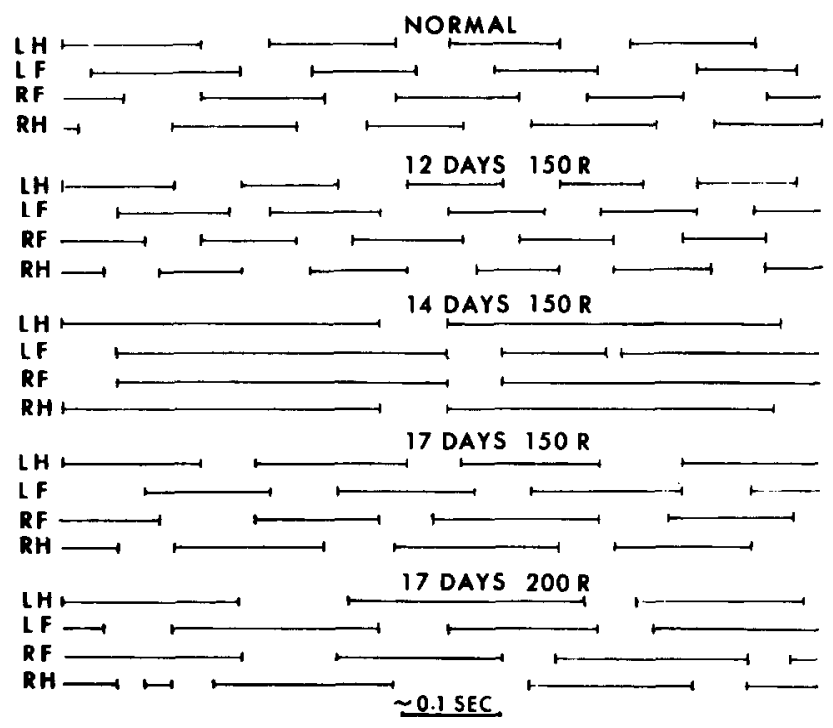

FIG. 2. Gait diagrams of prenatally irradiated rats traversing a narrow path (rectangular) compared with a normal rat. Lines represent stance times, the small vertical marks at each end indicating the beginning and end of a complete footfall. Spaces record swing time. The diagrams conventionally (16) begin with the left hind foot (LH). LF, RF, and RH indicate left and right fore and hind footfalls. The 12-day and 17-day 150-R animals are not distinguishable from normals. The 14-day rat hopped with hind and forelimbs. The 17-day, 200-R rat showed variable swing and stance times.

observed in carlicr experiments with 13-day irradiated rats (15).] There often was something of a unilaterally directed component to the act: The animal turned its forebody slightly to one side, and the hind foot on that side just contacted the animal's coat, while the other limb scratched aimlessly in space.

Jumping. All rats learned to jump 20 or $25 \mathrm{~cm}$ from one platform to another about as quickly as normal controls, except that the three just mentioned that had the most difficulty with locomotion jumped only 10 or $15 \mathrm{~cm}$. In such short jumps as the latter the forefeet reached the landing platform as the hind feet were about to leave the jumping platform. One of the latter animals, a 14-day rat, and two 15-day rats that hopped persistently with hind limbs, but could jump well, were tested with the landing platform inclined sideways about $40^{\circ}$. Their hind and forelimbs on each side independently adapted immediately to the slope, that is, the downside pair of limbs extended more to keep the animal upright. The 14-day rat making a short jump was clumsy, but did not fall off the landing platform. 
Righting. In movies of one normal rat, one 14-day rat that hopped with hind and forelimbs, and three 15-day rats that hopped with hind limbs, to observe righting, the animals were held so that they lay supine and quiet in the observer's hand with limbs partly extended upward. When released, the head and shoulders of the normal rat started turning simultaneously to one side toward a prone position, the forelimb on that side became a little more extended, then the other, then the trunk and hindquarters followed in the turning maneuver. At about two-thirds the distance to the ground, the animal was prone with limbs extended to land precisely on all fours. One 15-day rat did nearly as well, in the same style. The other three rats followed the same general style, but their limbs were unevenly poised for landing, and one 15-day and especially the 14-day rat usually landed a little off center or partly on one side of the body. (We were unsuccessful in testing tonic neck reflexes and consequent limb movements with the animals supine in a $V$ trough; though tame enough to lie still, they quickly stood when the head was turned.)

Transection of Spinal Cord. Hours after surgical transection of the spinal cord of mature rats, firm pinching of one hind foot elicited repeated exaggerated, irregular crossed extension reflex movements (mass reflex) in normal and hopping rats, and simultaneous pin-prick of the dorsa of both hind feet usually resulted in bilateral withdrawal in normal and in hopping rats. As the animals stabilized in a day or two, and during the periods of observation thereafter, normal rats usually responded to bilateral dorsal pin-prick with crossed extension reflexes, and hopping rats responded with bilateral simultaneous withdrawals, of the hind limbs. With about 10 repeated trials daily for a period of weeks, this pattern of crossed extension in normal rats and bilateral withdrawals in hopping rats was a consistent response pattern. (Pricking both plantar surfaces regularly resulted in bilateral withdrawal of the hind limbs in normal and hopping rats.) Pricking one foot elicited withdrawal of that foot, or crossed extension in normal animals, whereas in hopping rats it resulted in one or both feet being withdrawn, but occasionally a crossed extension reflex occurred. Firm pinching of, or closely repeated minor tactile stimuli to, one or both feet could set off the mass reflex during these later periods. These responses were often quite vigorous and sustained.

Functional Comparison with Other Rats. Hopping rats, as described previously, 17-day 150-R, and 12-day rats could all jump various distances ( 20 to $25 \mathrm{~cm}$ was the maximum usually tested) and directions essentially as well as normal, a manifestation of visually guided motor function. The 200-R, 17-day rats were not tested on jumping. Rats whose motor-sensory cortex was bilaterally ablated at birth usually did not jump from one platform to another, but stepped a short distance from the jumping platform 
to the landing platform when it was within reach (8). They learned to discriminate visual patterns as well as normal animals, but their rate of acquisition was slower. They had great difficulty traversing the rectangular narrow path, but locomotion on a broad path appeared virtually normal, although it was acquired more slowly than normally. Thus absence of the motor-sensory cortex interfered seriously with certain motor functions, but did not interfere with learning the sequences of movements necessary to perform the tasks, however clumsily they were executed (8). The 14-, 15-, and 17-day 150-R rats apparently had sufficient motor-sensory cortex and visual cortex to carry out visually guided jumping. In earlier experiments, as noted previously (4), 14-, 15-, and 17-day rats showed significant ability to discriminate visual patterns, functions that were regarded as cortically dependent in the experimental setting (13).

Gait diagrams (16) were prepared from the movies of a number of animals, on both the broad and the narrow rectangular pathways. These displayed the time that the four feet were on (stance phase) and off (swing phase) the ground (Fig. 2), the bars representing stance. The scale in Fig. 2, about $0.1 \mathrm{~s}$, corresponded to about 6.5 movie frames, at about 64 frames per second. For the work presented here, gait diagrams were a way of showing the interrelations of the four footfalls of the various rats in graphic comparison. Each gait diagram in Fig. 2 represents a train of consecutive locomotor limb cycles; even in the normal animals there were minor variations in the duration of the time the feet were on and off the ground. The diagram of the hopping rat corresponds to its real appearance, although plotting the gait diagram showed that it occasionally lifted one forefoot from the track momentarily (about one-sixtieth of a second) during the stance phase. The diagrams of the 12-day rat resembled those of normal rats. In the diagram of a 17-day, 150-R rat whose locomotion was close to normal, the footfalls were not much more variable than those of normal and 12-day rats. The record of the 17-day, 200-R rat showed substantial variability in the length of swing and stance time. Two hundred-roentgen rats and some $150-\mathrm{R}$ rats also showed a variation of length of stride as measured in the consecutive steps of the two hind limbs, as noted in the preceding paper (4). These faults undoubtedly underlie the lack of smoothness and altered cadence that the eye detected with ordinary observation and slow-motion movies. Variability was minimal in the hopping rats, odd as the gait was.

\section{Anatomy and Histology of the Spinal Cords}

Autoradiographic studies of normal cord development and some effects of radiation on developing cells are reviewed first, and a consideration of the cords of the mature irradiated rats follows. 
Autoradiographic Studies. Although there was a gradient of development in the cord in which the cervical region matured slightly before the lumbar region, it will be sufficient for most of our presentation here to generalize for the whole cord (Fig. 4). The first neurons laid down were large ventral horn motor neurons produced on the 12th day. No spinal cord neurons were generated in either of two litters labeled on the 11th day when, in our strains of rats, the cord was just becoming a tube. The motor neurons generated on the 12th day had, in less than $60 \mathrm{~h}$, migrated to their ventral lateral terminations and had become morphologically differentiated into young neurons. Some earliest posterior root ganglion neurons were also formed during this time. On the 13th day, many smaller neurons throughout the ventral horn, especially its more lateral aspects, and the middle lateral gray matter, were produced. A number of ventral horn motor neurons destined for the most ventral tip of the horn were also generated. A few posterior root ganglion neurons were formed.

Substantial numbers of cord cells generated on the 13th day were already at their destinations and morphologically differentiated into young neurons at the end of $48 \mathrm{~h}$ (on the 15th day). However, many heavily or moderately heavily labeled immature cells (hereafter, simply heavily labeled cells), beginning to resemble neurons, were still migrating in the intermediate zone between the central proliferative zone and more lateral part of the gray matter. In another sample from the same litter at $120 \mathrm{~h}$ (18th day) and in later samples to maturity, the regional distribution of the heavily labeled neurons was basically that which could be recognized during the first few days after H3T was given. Integration of the successive cell migrations of the 12th and 13th days was observed. After generation of a majority of the large motor neurons (Rexed's lamina IX) during the 12th day, many smaller neurons forming parts of Rexed's lamina VII, which makes up much of the ventral horn and central and lateral gray matter, were formed on the 13th day and migrated to partly surround the large motor neuron assemblies. Simultaneously, another small cohort of large motor neurons was produced to migrate with the smaller VII cells to their position in the ventralmost tip of the ventral horn as another component of lamina IX.

On the 14th day the distribution of generated neurons widened, and they were generally well established at their terminations by $72 \mathrm{~h}$. A substantial number found their way to the medial ventral gray, corresponding to Rexed's VIII and the medial more dorsal part of VII. The greatest number of neurons produced on day 14 were those destined for the dorsal horn, quite widespread through it. Dorsal root ganglia neurons were produced in larger numbers than on day 13 . Very rarely, a heavily labeled large ventral horn neuron was encountered, the last of that component to be produced.

On the 15th day most migrations of neurons-to-be were to the dorsal 
horns, especially their lateral parts, with fewer to the middle gray dorsomedial part of the ventral horns. Cells generated on the 16th day included some neurons destined for the dorsal horns, and on the 17th day very few neurons were generated, the principal products being ependymal cells and other neuroglia to keep pace with cord growth. Mitotic activity continued through the $21 \mathrm{st}$ day, just before term, in the ependymal and subependymal regions dorsally, laterally, and ventrally. Thereafter, growth was regarded as being the maturation of already formed elements for several months, and a subsiding multiplication of glia to a steady low rate of turnover for the animal's lifetime.

Certain G2 (late prophase) and G1 (postmitotic primitive proliferative and primitive differentiating) cells surrounding the central canal of the cord (and in other parts of the fetal central nervous system) showed selective vulnerability to being killed quickly by 150 to $200 \mathrm{R}$ as determined by irradiating at specified times after the $\mathrm{S}$ (synthesis) phase cells took up H3T $(3,12,14)$. In a 12-day embryo some or most of the products of proliferation during the labeling process had migrated, arrived at their destinations, and differentiated into neurons within 2 days. Irradiation would kill many of the proliferative cells as well as many of their products, the migratory cells, that were still in a primitive stage and vulnerable to radiation. Similarly on the 13 th, 14th, and 15th days, proliferative cells and primitive differentiating migratory cells were vulnerable. At a particular stage, then, say mid-14th day, not only were many proliferative cells destroyed, but also many other cells were killed that had been produced a day earlier, but which were still primitive enough to be vulnerable as they migrated to their destinations. The cell killing that occurred in such circumstances during embryonic and fetal life (irradiation with 150 or $200 \mathrm{R}$ ) virtually never failed to be counterbalanced in a lesser or greater degree by the regulative capacity of the residual proliferative cells to restitute the damage. In the 12th-day cord this capacity was great, and a remarkable degree of recovery occurred, as we noted, visibly beginning in less than $9 \mathrm{~h}(4,5,12,14$; D'Amato and Hicks, unpublished observations). In the 13-, 14-, and 15-day cords, the dorsal half of the proliferative zone failed noticeably in its regulative, regenerative effort, and the cells formed rosettes, that is, abnormal centers of proliferative cells. It was more difficult to determine if there was a measurable loss of cells in the ventral horns on those days - and on the 12th day - as we consider in the following.

Anatomic Findings. The normal adult spinal cord of our rats, in the fresh state, was about 4.5 to $5 \mathrm{~mm}$ wide in the lower midcervical region (about $\mathrm{C} 4$ to $\mathrm{C6}$ ) and about $4.5 \mathrm{~mm}$ at $\mathrm{L} 1$, the widest portions. These dimensions were a little reduced in some but not all 12-day and 17-day rats. They were usually reduced in 14- and 15-day rats. The cords of five 12-day, seven 

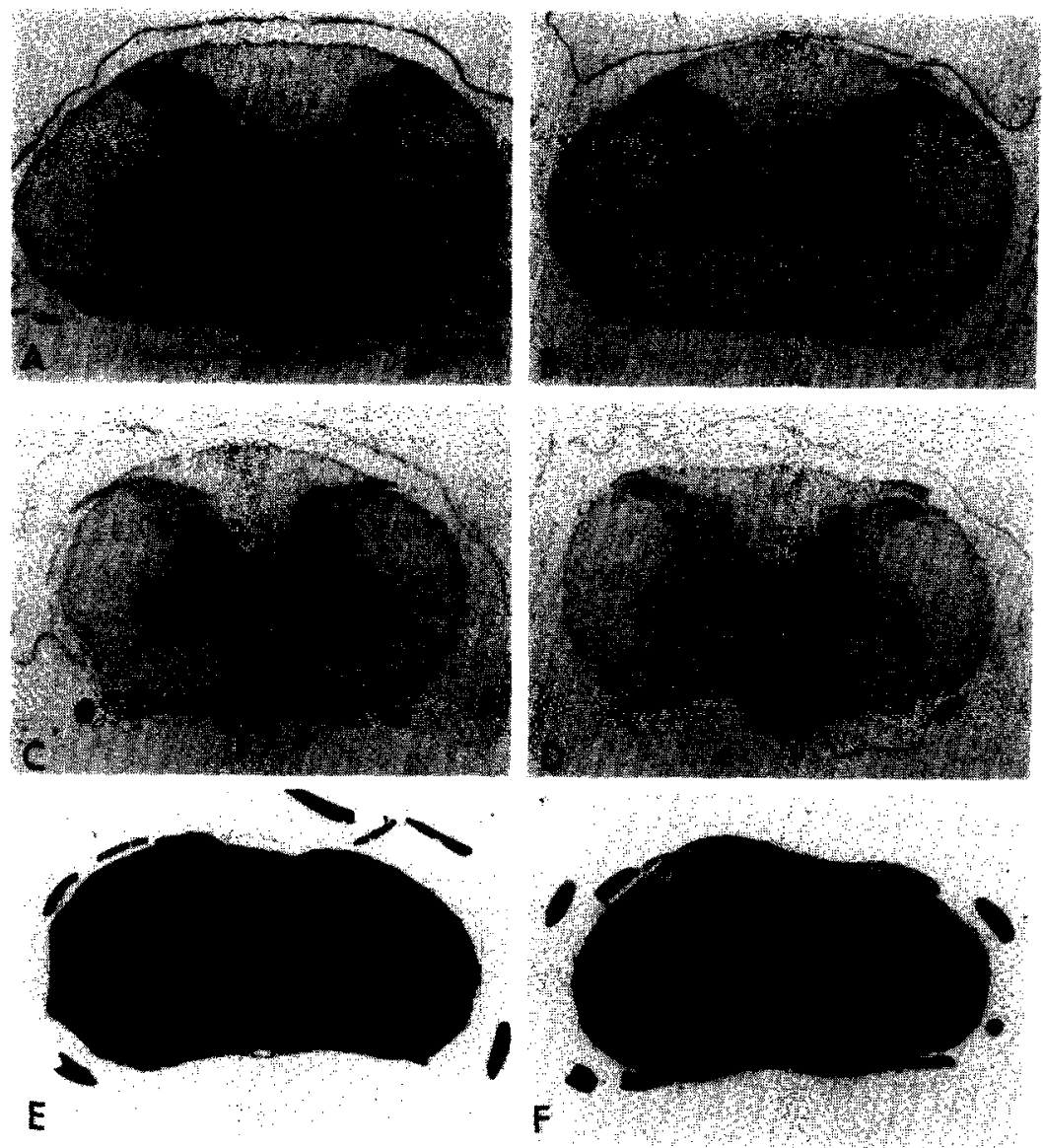

FIG. 3. Cross sections of the cervical spinal cords, between $\mathrm{C} 4$ and $\mathrm{C5}$, of four prenatally irradiated rats compared with two normal rats. A-normal. B-150 R, 12th day, nearly normal, with a slight overall reduction of gray matter. $\mathrm{C}-150 \mathrm{R}, 14$ th day shows reduction of the posterior horns $(\mathrm{PH})$ and diminished cross section. $\mathrm{D}-150 \mathrm{R}, 17$ th day shows some overall reduction. Cresyl violet. E-normal. F-150 R, 15th day shows thickening of the gray commissure region and reduction of the posterior horns, but virtually no reduction of the anterior horns. Luxol fast blue-cresyl violet.

14-day, four 15-day, four 17-day rats, and five normal rats were examined histologically at cervical, thoracic, lumbar, and usually sacral levels. In Fig. 3 the cords of normal rats are compared with those of 12-, 14-, 15-, and 17-day rats. A, B, C, and D are from levels between $\mathrm{C} 4$ and $\mathrm{C5}$, at the beginning of the intumescence, where the cord begins to change in cross section from oval to ventrally flattened oval shape. B, 12-day, shows a slight overall reduction of gray matter. Some of the difference in the total 
size may be owing to the slightly more caudal level of the normal, A. The smaller cord of the 17-day rat, $\mathrm{C}$, whose locomotion was very nearly normal, is difficult to explain. A few cells destined for Rexed's laminae I and II were killed which would be difficult to recognize in the form of a deficit. The animal was substantially smaller than normal, and that, with the slight difference of cord levels between $\mathrm{C}$ and $\mathrm{A}$, might account for some of the difference. Radiation in the doses used had some permanent effect on the differentiation of young neurons, and as little as 10 to $20 \mathrm{R}$ could permanently alter early dendritic growth in some circumstances, but these effects were not ordinarily easy to detect $(10,13)$. The cords of some 17-day rats were essentially normal in appearance irrespective of their motor behavior. The cords of $14-$ and 15-day rats were usually a little smaller in diameter than normal, in one instance fairly substantially smaller. There were two major findings. One was a thickening and sometimes cytoarchitectural distortion of the central gray commissure region, including Rexed's laminae V and VI, and the dorsal margin of VII that lay in

$\begin{array}{ccccc}\text { STAGES } & \text { CORD } & \begin{array}{c}\text { CORD } \\ \text { ABNORMALITIES ASSOCIAIED }\end{array} & \text { MAJOR } \\ \text { OF CORD } & \text { NEURONS } & \text { PRODUCED BY } & \text { BRAIN } & \\ \text { DEVELOPMENT } & \text { GENERATED } & \text { I5OR } & \text { ABNORMALITIES } & \text { LOCOMOTION }\end{array}$

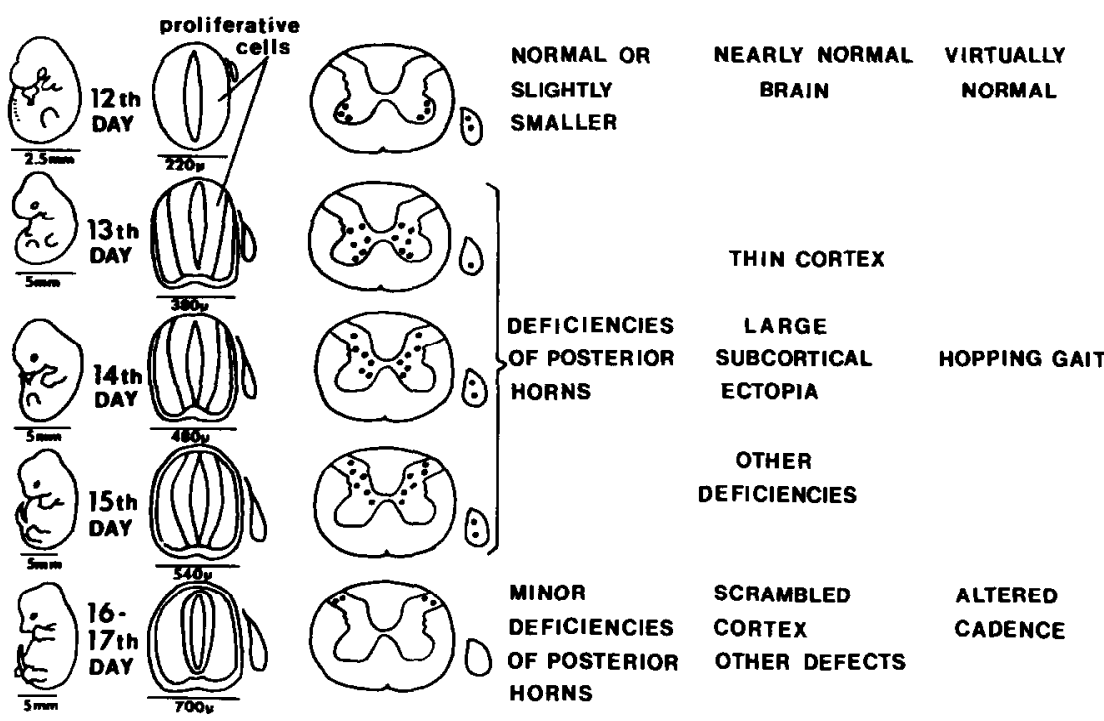

FIG. 4. Summary diagram of the development of neurons in the rat's spinal cord, based on H3T autoradiographic studies, and principal consequences of $150 \mathrm{R}$ on fetal days 12 to 17 . Dots in the spinal cords and their ganglia indicate the neurons generated each day. The diminishing proliferative cell zone around the central canal is shown in the second column, and corresponding fetal stages are drawn in the first column. 
the base of the dorsal horn. This resulted from the original destruction and subsequent rosette formation after irradiation and is shown in Fig. 3F, a 15-day rat compared with a normal, E, at $\mathrm{L} 1$. The other major finding was a diminution of the entire posterior horn, especially evident in the head portion, and as a deficiency of the dorsomedial part of the neck portion, seen in Figs. $3 \mathrm{C}$ and F. Another demonstration of these abnormalities appears in Fig. 3 of the preceding paper (4). More difficult to determine was whether or not there was a significant reduction of the ventral horns. In 14-day rats, this would be possible, for as Fig. 4 shows, neurons destined for the dorsomedial parts of the ventral horns were being generated then, and cells produced the day before, still in transit to their ventral horn destinations, were vulnerable to radiation. In 15-day rats, the losses owing to radiation were limited to the posterior horns and only those dorsomedial parts of the ventral horn near the gray commissure, as may be inferred from Fig. 4. In Fig. 3F the ventral horns appear to be unaffected. The observation was significant in our attempts to explain how the morphologic changes in these rats caused the hopping gait, as we discuss later.

The CS tract was variably diminished, and sometimes irregular in its medullary decussation, in 14- and 15-day rats, diminished in 17-day rats, and nearly normal in size in 12-day rats.

\section{DISCUSSION}

The spinal cord transections provide the strongest evidence that the hopping gait is primarily caused by an abnormality in the spinal cord. That supraspinal mechanisms exercised governance of the hopping mechanisms, and adapted to them, was indicated by the animals' ability to jump various short distances from one horizontal platform to another, gauging the distance and direction visually. Also, if the landing platform was tilted laterally about $40^{\circ}$, the limbs were independently adjusted to keep the animal upright, even in severely affected subjects, though not as well. The gait was rapidly adapted to walking on horizontal ladders. Animals that retained hopping of the forelimbs tactually and visually placed independently with either limb. These mechanisms did not, however, override the hopping pattern in ordinary terrestrial locomotion and swimming, or using both hind limbs in scratching reflexes. Elements of the corticospinal, vestibulospinal, reticulospinal, and rubrospinal systems were shown by the HRP method to have developed. As we noted, the corticospinal tract in the cord in dye-stained sections was smaller than normal, but it attained the lumbar level.

It is difficult to correlate the anatomic deficiencies and possible disordered circuitry of the posterior horns with the hopping gait. 
Physiologic observations and conceptual models of the normal spinal locomotor generator of alternating gait emphasize circuits concerned with ventral motor neurons, closely associated ventral horn interneurons, and afferent input from muscles. Cutaneous and some other afferent impulses modify the basic function of the generator, and their circuitry involves interneurons in the dorsal horns. Though it was difficult to recognize, there could have been a deficit of ventral horn neurons in the 14-day rats as shown in the H3T autoradiographic and other studies of the developing cord, but a ventral horn deficit of interneurons, except of some close to the central canal region, could not have occurred in any appreciable degree in 15 -day rats according to these studies. We could come to no conclusions about the possible significance of spinal ganglion neurons that may have been lost. Of the seven 14-day rats and four 15-day rats whose cords were studied in detail thus far, those that hopped with all four feet had the most overall deficient posterior horns and this was combined with a thickening of the gray commissural region and bases of the dorsal horn. Rats that switched to a normal or nearly normal gait of the forelimbs, but not the hind limbs in the juvenile period, and the rat that switched to a normal gait nearly completely, had posterior horn defects but little or no central thickening. This group included 15-day rats.

The apparent discrepancy between the site of major anatomical changes in the posterior horns and the location of neurons thought to be the substrate of the locomotor generator in the ventral horns indicates that more information is needed before there is an explanation of the hopping gait. The puzzle is compounded by the partial switching that many animals made from a hopping to an alternating gait and the adaptations that the brain apparently made to control the hopping gait. Morphologic studies of fetal and infant cords and spinal ganglia where more nearly quantitative estimates of cell populations seem possible and studies of the morphology of the CS tract may help.

\section{REFERENCES}

1. Carter, D. A., AND H. C. Fibiger. 1978. The projections of the entopeduncular nucleus and globus pallidus in rat as demonstrated by autoradiography and horseradish peroxidase histochemistry. J. Comp. Neurol. 177: 113-124.

2. D'Amato, C. J., AND S. P. Hicks. 1978. Radiation effects on developing corticospinal neurons. Abstracts of VIIIth International Congress of Neuropathology, Washington, D.C., No. 74, p. 605.

3. D'Amato, C. I., AND S. P. Hicks. 1978. Normal development and posttraumatic plasticity of corticospinal neurons in rats. Exp. Neurol. 60: 557-569.

4. D Amato, C. J., AND S. P. Hicks. 1980. Development of the motor system: effects of radiation on developing corticospinal neurons and locomotor function. Exp. Neurol. 70: $1-23$. 
5. Hicks, S. P., B. L. Brown, AND C. J. D'AmATo. 1957. Regeneration and malformation in the nervous system, eye, and mesenchyme of the mammalian embryo after radiation injury. Am. J. Pathol. 33: 459-481.

6. Hicks, S. P., AND C. J. D'AmATo. 1966. Effects of ionizing radiations on mammalian development. Pages 195-250 in D. H. M. Woollam, Ed., Advances in Teratology. Logos Press, London.

7. Hicks, S. P., AND C. J. D'Amato. 1968. Cell migrations to the isocortex in the rat. Anat. Rec. 160: 619-634.

8. Hicks, S. P., AND C. J. D' Amato. 1975. Motor-sensory cortex-corticospinal system and developing locomotion and placing in rats. Am. J. Anat. 143: 1-42.

9. HICKs, S. P., AND C. J. D' AMATO. 1976. Labeling corticospinal and brain-stem neurons in mature, infant, and radiation-malformed rats by retrograde axonal transport of horseradish peroxidase. Abstr. Proc. Annu. Meeting Mich. Chap., Soc. Neurosci., Ann Arbor, Mich.

10. Hicks, S. P., AND C. J. D' Amato. 1978. Effects of ionizing radiation on developing brain and behavior. Pages 35-72 in G. GotTlieB, Ed., Studies on the Development of Behavior and the Nervous System: Early Influences. Academic Press, New York.

11. HICKS, S. P., AND C. J. D'AMA TO. 1979. Hopping locomotion in rats irradiated in utero. Soc. Neurosci. Abstr. 5: 116.

12. Hicks, S. P., C. J. D' Amato, M. A. Coy, E. D. O’Brien, J. M. Thurston, and D. L. JOFTES. 1961. Migrating cells in the developing nervous system studied by their radiosensitivity and tritiated thymidine uptake. Pages 246-261 in Symposium on Fundamental Aspects of Radiosensitivity. Brookhaven National Laboratory, Upton, N.Y., Office of Technical Services, Department of Commerce, Washington, D.C.

13. Hicks, S. P., C. J. D'Amato, and J. L. Falk. 1962. Some effects of radiation on structural and behavioral development. Int. J. Neurol. 3: 535-548.

14. Hicks, S. P., C. J. D'Amato, AND D. L. JofTes. 1962. The nature of the radiosensitive cells in the developing nervous system studied with tritiated thymidine. Pages 199-205 in B. Gross AND V. ZelenY, Eds., Effects of lonizing Radiation on the Nervous System. IAEA, Vienna.

15. Hicks, S. P., C. J. D' AmATo, AND M. J. Lowe. 1959. The development of the mammalian nervous system. J. Comp. Neurol. 113: 435-469.

16. Hildebrand, M. 1976. Analysis of tetrapod gaits: general considerations and symmetrical gaits. Pages 203-236 in R. M. Hermann, S. Grillner, P. S. G. Stein, and D. G. Stuart, Eds., Neural Control of Locomotion.-Plenum Press, New York.

17. Hruska, R. E., and E. K. Selbergeld. 1979. Abnormal locomotion in rats after bilateral intrastriatal injection of kainic acid. Life Sci. 25: 181-194.

18. Mullenix, P., S. Norton, And B. Culver. 1975. Locomotor damage in rats after X-irradiation in utero. Exp. Neurol. 48: 310-324.

19. NieuwenhuYs, R. 1976. Aspects of the morphology of the striatum. Pages 1-17 in A. R. Cools, A. H. M. Lohman, and J. H. L. van Den Bercken, Eds., Psychobiology of the Striatum. North-Holland, Amsterdam.

20. Nornes, H. O., AND G. D. DAS. 1974. Temporal pattern of neurogenesis in spinal cord of rat. I. An autoradiographic study - time and sites of origin and migration and settling patterns of neuroblasts. Brain Res. 73: 121-138.

21. REXED, B. 1964. Some aspects of the cytoarchitectonics and synaptology of the spinal cord. Pages 58-92 in J. C. ECCLES AND J. P. SCHADE, Eds., Organization of the Spinal Cord. Elsevier, Amsterdam. 